http://jmscr.igmpublication.org/home/ ISSN (e)-2347-176x ISSN (p) 2455-0450 crossref DOI: https://dx.doi.org/10.18535/jmscr/v8i4.22

\title{
A Study of Patient's Perception about the role of Anesthesia and Anesthesiologist
}

\author{
Authors \\ Umar Jan $^{1}$, Ajaiz Rasool ${ }^{*}$, Showkat A Gurcoo², Sabina Nisar ${ }^{3}$ \\ ${ }^{1}$ Senior Resident, Anaesthesiology, ${ }^{2}$ Professor, Anaesthesiology, ${ }^{3}$ Senior Resident, Surgery \\ Sher-I-Kashmir Institute of Medical Sciences, Soura Srinagar Kashmir \\ *Corresponding Author \\ Dr Ajaiz Rasool
}

Senior Resident, Sher-I-Kashmir Institute of Medical Sciences, Soura Srinagar Kashmir, India

\begin{abstract}
Background: To assess the perception about anesthesia and role of anesthesiologists among lay communities, as anesthesiology has been considered as behind the scene specialty and image of anesthesiologist has always been problem.

Design: Self-structured questionnaire evaluation

Methods: This prospective questionnaire based study was conducted in SKIMS-Soura among 150 randomly selected patients, admitted for elective surgeries.

Results: Mean age was 25.33 years and majority of them were females. $81 \%$ of respondents knew that anesthesiologist is a doctor and $49.5 \%$ knew that anesthesiology is a different specialty; there was significant association between education level of respondents and this response. Of the respondents $77 \%$ felt that that anesthesiologist was easy to talk and pleasant by the bedside. Only $37.3 \%$ of respondents considered some role of anesthetist in postoperative period. $31 \%$ of respondents only were satisfied with explanation of anesthesiologists regarding method of procedure, options and possible complications.

Conclusion: Patients still have inadequate knowledge regarding anesthesia services and role of anesthesiologists.
\end{abstract}

\section{Introduction}

Anesthesiology is one of the youngest branches of medical science that has made immense development. Role of anesthesiologist has extended not only inside but also outside traditional operating room settings.

Anesthesiologists and anesthesia has always been considered as behind the scene specialty. The image and status of anesthesiologist in the eyes of medical and lay communities has always been problem $^{1}$. The widespread role of anesthesiologist in the short term care, as well as in pain relief, is not known to the public, and they have very limited knowledge about the specialty ${ }^{2}$. Although there is tremendous health care awareness, still there is hardly any knowledge of the medical services and practices relating to operating room and to anesthetic management. Although it is not known whether it will be of any further benefit to make the public much more aware of specialty, surveys have been conducted throughout the world to assess the public perception of 
anesthesiologist $^{3-7}$. With this background, we conducted a study in SKIMS in an attempt to discover the knowledge of patients regarding role of anesthesia and anesthesiologist.

\section{Methods}

The study was conducted in SKIMS after obtaining approval from hospital ethical committee. Study was purely oral in nature. It was structural interview based on self-structured questionnaire. Interviews were carried out by team of anesthesiologists during preoperative visit. 150 patients scheduled for various elective surgeries were included randomly in the study. Patients with age less than 15 years, severe medical illness, inability to speak, brain damaged, refusal to participate, were excluded from the study. Questionnaire had questions in total, inquiring about patient's knowledge of role, presence of anesthesiologist inside theatre, concerns and complicationsetc.

\section{Results}

1. Demographic Profile: The mean age of the respondents was 25.33 years and majority of respondents were females (M: F- 54:96).

We distributed respondents based on educational level, and majority of patients belonged to primary and high school level. There was significant relationship between the level of education and the knowledge of patients regarding that anesthesia is a different specialty.

\begin{tabular}{|l|c|c|}
\hline $\begin{array}{l}\text { Education } \\
\text { level }\end{array}$ & $\begin{array}{c}\text { Number } \\
(\boldsymbol{\%})\end{array}$ & $\begin{array}{c}\text { Percentage who } \\
\text { knew anesthesia is } \\
\text { different specialty }\end{array}$ \\
\hline None & $36(24 \%)$ & $40 \%$ \\
\hline Primary & $41(27.3 \%)$ & $56 \%$ \\
\hline Secondary & $51(34 \%)$ & $65 \%$ \\
\hline Graduation & $16(10.6 \%)$ & $81 \%$ \\
\hline University & $6(4 \%)$ & $94 \%$ \\
\hline
\end{tabular}

2. Role of anesthesiologist: $81.3 \%$ of respondents knew that anesthesiologist is a doctor and $49.4 \%$ of respondents had knowledge that anesthesiology is a different specialty.

3. Work of anesthesiologist in OR: $66.3 \% \mathrm{OF}$ respondents knew that anesthesiologist has a definite role in the operating room, however, most of them thought that the role was limited to administering gases and monitoring patients intraoperatively. Only $11.3 \%$ respondents has added information about the role of anesthesiologist.

4. Role of anesthesiologist in postoperative period: Out of the respondents who knew anesthesiologist has definite role, only $37.3 \%$ of respondents had knowledge that anesthesiologist are involved in immediate post-operative care and complications, and $19.2 \%$ knew that anesthesiologist manage pain and rest did not know anything.

5. Knowledge about different kinds of anesthesia: Most of respondents (71.2\%) knew that anesthetic techniques consisted of general anesthesia only, $11.3 \%$ had idea also about regional anesthesia techniques and rest of respondents did not have any idea about techniques of anesthesia.

6. Knowledge about regional anesthesia: Among the respondents, who knew about regional anesthetic techniques, $26 \%$ had knowledge about spinal anesthesia and $41.7 \%$ of respondents knew about local infiltration, and rest had no idea.

7. Post-operative complications: about $36 \%$ of respondents had some knowledge about anesthetic complications and rest majority of patients did not have any knowledge about complications of anesthesia.

8. Attitude of anesthesiologist: when asked about the bed side manner of anesthesiologist, about $77 \%$ of respondents revealed pleasant bedside manner of anesthesiologist. $31 \%$ of respondents were satisfied by the explanation and discussion by anesthesiologists about methods, options to put on sleep and about possible side effects of drugs and possible complications. 


\section{Discussion}

Regarding perception of patients about anesthesiologists and anesthesiology, various reports have been published previously and overall, there has been little change in the knowledge among general population regarding the specialty ${ }^{8}$. The reason for this may the less contact of anesthesiologist with conscious patient than other specialists ${ }^{9}$. The modern medical era requires expertise of anesthesiologist than any other medical specialty, acquiring role of "perioperative physician".

In the present study, around $80 \%$ of respondents knew that anesthesiologist was a doctor compared with $67 \%$ and $65 \%$ in two surveys done in UK in 1978 and 1994, respectively ${ }^{10,11}$. This might be due to introduction of pre-anesthetic clinics, routine preoperative and postoperative visits combined with increasing awareness among patients.

It is heartening to note that more than $70 \%$ of respondents felt that anesthesiologist and pleasant manners and easy to talk. Even a previous study has found that attire of anesthesiologist can affect perception of patient ${ }^{12}$. Many of the respondents, however, were not satisfied with the explanation from anesthesiologist regarding the procedure of anesthesia, possible side effects and complications. Previous studies have also found that anesthesiologists failed to explain the details of anesthesia to the patients ${ }^{13}$. The possible explanation for this may be that many anesthesiologists did not want to disclose every detail for fear of increasing patient anxiety, and many patients accept the paternalistic attitude of physicians $^{14,15}$.

The questions regarding about role of anesthesiologist has mixed response. Many of the respondents knew that anesthesiology is a different specialty, however, in present study, significantly limited number of respondents knew about spectrum of services provided by anesthesiologists, different techniques and possible side effects and complications of anesthesia. This is in concordance with previous studies ${ }^{16}$.

In our study the knowledge of patients about anesthesiology and anesthesiologist correlated well with level of academic qualification. Previous studies have also found that patients with better academic qualification had better knowledge of anesthesia and anesthesiologist. ${ }^{17}$

Broadly our study revealed limited knowledge of anesthesia and anesthesiologist as a specialty among general masses. The matter of debate is that whether public image of anesthesiologist is really important. Some authors have opinion that anesthesiologists should not be concerned to widespread public ignorance of specialty ${ }^{18}$. Other authors have argued that increasing appreciation by public will increase the ability to influence the provision of resources for the specialty ${ }^{19}$. The lack of recognition can affect the self-esteem of anesthesiologist and amount of effort required to achieve expertise $\mathrm{e}^{20}$. There should be some means to disseminate information regarding the widespread role of anesthesiologist inside and outside operating rooms, including pain clinics, emergency medicine, ICU.

In summary our study reiterates poor knowledge of specialty among general population and highlights the need for exposure of the specialty to patients, making use of media and internet to improve image in the eyes of the public.

Acknowledgement: We are highly thankful to the patients of SKIMS who have cooperated and contributed significantly to the accomplishment of study.

\section{References}

1. Armitage EN. The public image of the specialty \{letter\}. Anaesthesia. 1978 Jan;33(1):64-5.

2. Simni B. Anesthetist: the wrong name for the right doctor. Lancet 2000May 27;355(9218): 1892

3. Pleuvry BJ, Bradshaw EG. The anesthetist in the eyes of the public. Anaesthesia 1982;37:462-3. 
4. Van Wijk MGF, Smalhout B. A postoperative analysis of the patient's view of Anaesthesia in a Netherlands teaching hospital.AnesthAnalg 1991; 73:190-8.

5. Sheved K, Pnagopoulos G. A survey of 800 patient's knowledge, attitudes and concerns regarding Anaesthesia. Anesth Analg 1991;73:190-8.

6. Chew ST, Tan T, Tan SS,Ip-Yam PC. A survey of patient's knowledge of Anaesthesia and perioperative care. Singapore Med J 1998;39:399-402.

7. Calman LM, Mihalache A,EvronS, Ezri T. Current understanding of the patient's attitude towards the anesthetist roleand practice in Israel: effect of patients experience . J ClinAnesth 2003;15:451-4.

8. Bailard C, Lamberto JF, Martinez C, et al. Patient's perspective with regard to anesthesia services. Can J Anaesth 2001;48:526-8

9. Hume MA, Kennedy B, Asbury AJ. Patient knowledge of Anaesthesia and perioperative care. Anaesthesia 1994; 49:715-8

10. Keep PJ, Jenkins JR. As others see us. The patient's view of the anaesthetist. Anaesthesia 1978; 33:43-5.

11. Swinhoe CF, GrovesER. Patient's knowledge of anesthetic practice and the role of anesthetic. Anaesthesia 1994; 49:165-6.

12. Hennessy N, Harrison DA, AitkenheadAR. The effect of the anaesthetist's attire on patient attitudees. Anaesthesia 1993; 48:219-22

13. Ali S, Vivekanandan P, Tierney E. Patients perception of anaesthetist and Anaesthesia. Anaesthesia 1994;49:644-5.

14. Jafarey AM, Farooqui A. Informed consent in the Pakistani milieu: the physicians perspective. J Med Ethics 2005;31:93-6.
15. Stiggelbout AM MolewijkAC,Otten W, Timmermans DR, van Bockel JH, Kieviot J. Ideals of patient anatomy in clinical decision making: a study on the development of a scale to assess patients and physician views, $\mathbf{J}$ Med Ethics 2004;30:268-74.

16. Bhattarai B, Kandei S, Adhikari N. Perception abouit the role of anesthesia and anesthesiologist among the paramedical staff: perspective from medical college in Nepal. Kathmandu Univ Med J 2012; 38(2):51-54.

17. Calman LM, Mihalache A, Evron S, Ezri T. Current understanding of the patient's attitude toward the anesthetist's role and practice in Israel: effect of the patient's experience. J CLIN Anesth. 2003sep; 15 (6):451-4.

18. Hall IA, Earlam C. They are not proper doctors, are they? Anaesthesia 1995;50:87. 